\title{
An ultra-thin PDMS membrane as a bio/micro-nano interface: fabrication and characterization
}

\author{
Abel L. Thangawng • Rodney S. Ruoff • \\ Melody A. Swartz • Matthew R. Glucksberg
}

Published online: 22 May 2007

(C) Springer Science + Business Media, LLC 2007

\begin{abstract}
We report a method for making ultra-thin PDMS membrane devices. Freely suspended membranes as thin as $70 \mathrm{~nm}$ have been fabricated. Bulging tests were performed with a custom built fluidic cell to characterize large circular membranes. The fluidic cell allows the media (such as air or water) to wet one side of the membrane while maintaining the other side dry. Pressure was applied to the membrane via a liquid manometer through the fluidic cell. The resulting load-deflection curves show membranes that are extremely flexible, and they can be reproducibly loaded and unloaded. Such devices may potentially be used as mechanical and chemical sensors, and as a bio-nano/micro interface to study cellular mechanics in both static and dynamic environments.
\end{abstract}

Keywords Microfabrication P PDS membrane ·

Bulging test $\cdot$ Ultra sensitive membrane

\section{Introduction}

Soft elastic substrates are often used in biological experiments to study cell locomotion, mechanical/traction forces, and the organization of focal adhesions of cells, among many other topics (Harris et al. 1980; Burton and

\footnotetext{
A. L. Thangawng $\cdot$ R. S. Ruoff

Mechanical Engineering Department,

Northwestern University, Evanston, IL, USA

M. A. Swartz $\cdot$ M. R. Glucksberg $(\square)$

Biomedical Engineering Department,

Northwestern University, Evanston, IL, USA

e-mail: m-glucksberg@northwestern.edu
}

Taylor 1997; Balaban et al. 2001). Polydimethylsiloxane $(\mathrm{PDMS})^{1}$ in particular has become a preferred soft substrate due to its biocompatibility and ease of processing (Peterson et al. 2005; Ostuni et al. 2000; De Silva et al. 2004; Tan et al. 2003; du Roure et al. 2005). PDMS can also be easily processed to have a range of Young's modulus values that are physiologically relevant (Brown et al. 2005). Several recent cellular mechanics studies (Lo et al. 2000; Wang et al. 2001; Bischofs and Schwarz 2003; Bischofs et al. 2004) were conducted using a thick ( $\sim 50 \mu \mathrm{m}$ or thicker) sheet of the soft polymeric substrates, PDMS or polyacrylamide, directly placed on a coverslip. Since we are interested in studying the cell-substrate interaction in a mechanically dynamic environment, we have developed freely suspended PDMS membrane devices that are extremely flexible due to both the very low Young's modulus and the thickness of the PDMS.

Here, the design and fabrication of such PDMS membrane devices, and the characterization of the larger membranes (a diameter of $300 \mu \mathrm{m}$ or larger) are described. A fluidic cell was also designed that allows a desired media to be supplied on one side of the membrane while the other side is maintained in the dry state for imaging and/or other engineering/interfacing purposes. Several membranes were characterized by performing bulging test using the fluidic cell (using both air and water as the pressure media). The membrane's extreme flexibility makes it suitable for further development of ultra sensitive membrane devices in the future.

\footnotetext{
$\overline{{ }^{1} \text { Sylgard } 184}$, Dow Corning.
} 


\section{Device fabrication}

\subsection{PDMS membrane device fabrication}

The fabrication of the PDMS membrane devices involves parallel processing of multiple parts that are assembled at appropriate stages. Two different types of devices were fabricated: (1) devices that have membrane thickness larger than $\sim 500 \mathrm{~nm}$ with a diameter ranging from a few hundred micrometers to $2 \mathrm{~mm}$, and (2) membranes with a thickness between 70 and $500 \mathrm{~nm}$ for smaller diameter membranes (of a few micrometers). We have earlier reported a technique for making PDMS membranes as thin as $70 \mathrm{~nm}$ (Thangawng et al. 2007; Thangawng and Lee 2004). Figure 1 shows the process flow for fabricating a freestanding ultra-thin PDMS membrane device. For the larger and thicker membrane devices, the steps related to making a perforated membrane as shown in Fig. 1 do not have to be included in the fabrication process. In that case, the membrane can be directly bonded on the patterned PDMS structure. If desired, micro/nano patterns can also be incorporated into either side of the PDMS membrane by introducing the patterns on the mold (before curing) (Thangawng and Lee 2004), or by directly imprinting the patterns (during the curing of the flat membrane) (Kim et al. 2001; Khang and Lee 2004). Both the top and bottom surfaces of the large membranes are accessible for interaction with the systems being studied in these devices, and also for integrating other components or devices with the system.

To achieve extremely flexible and sensitive membranes, PDMS was diluted with hexane (ACS grade, Fisher Scientifics), which allowed the fabrication of membranes

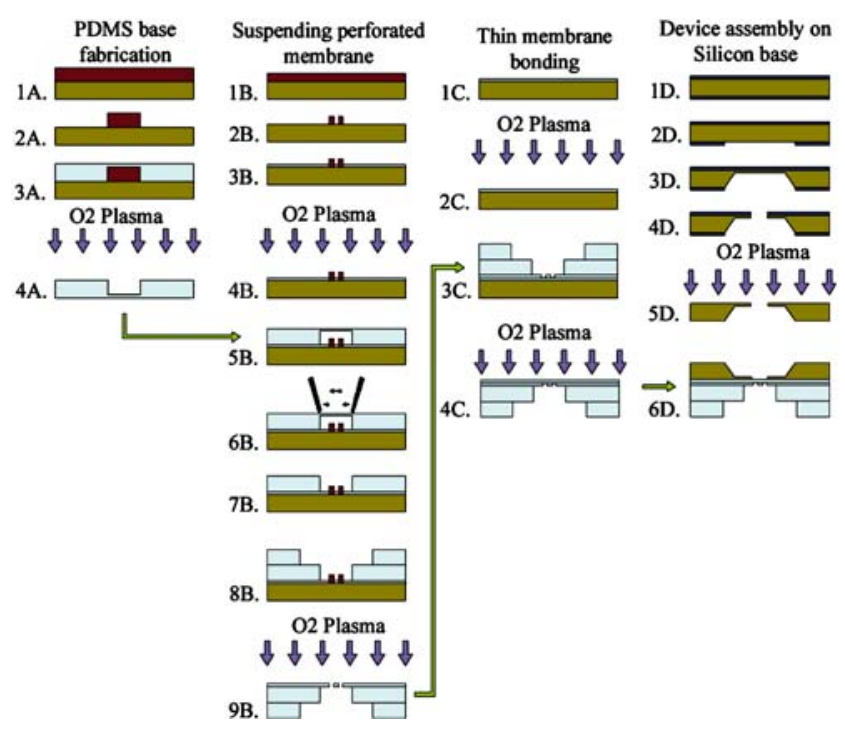

Fig. 1 Fabrication process for ultra-thin PDMS membrane

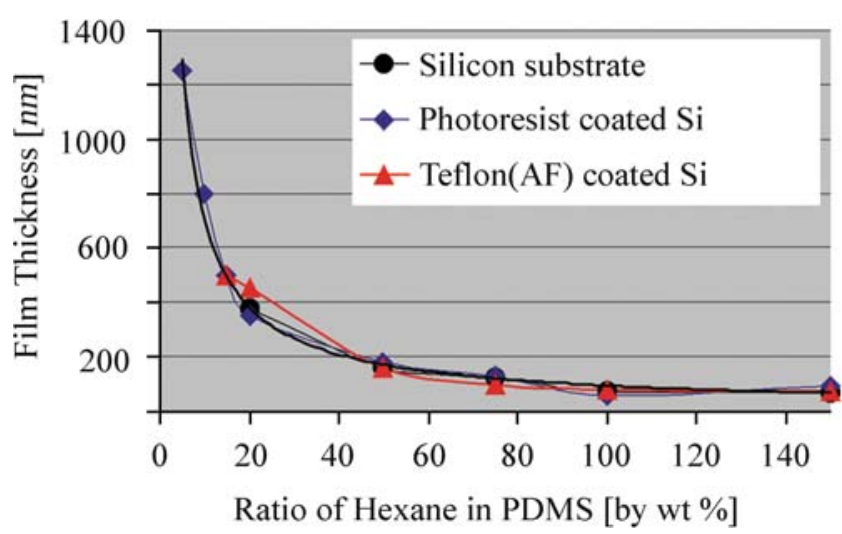

Fig. 2 PDMS film thickness on three different substrate types for different ratios of dilution with hexane

as thin as $\sim 70 \mathrm{~nm}$. In all our sample preparations, a PDMS pre-polymer to curing agent ratio of 10:1 was used. The membrane thickness as a function of the ratio of dilution of PDMS in hexane is shown in Fig. 2. The spin rate and duration were kept constant at 6000 RPM and $150 \mathrm{~s}$, respectively. We used the Bond-Detach Lithography technique (Thangawng et al. 2007) to partially remove the PDMS film from the substrate, creating an edge that exposes the full cross-section of the film. An AFM (MultiMode SPM; Digital Instruments) or optical profilometer (MicroXam, Phase Shift Technology) was used to measure the film thickness. Without diluting the PDMS, the thickness of the membrane could only be reduced down to $\sim 3 \mu \mathrm{m}$ (Lotters y et al. 1997).

\subsubsection{PDMS base fabrication}

For membranes with a lateral dimension of $100 \mu \mathrm{m}$ or larger, a thick SU8-2100 photoresist (MicroChem Corp) was first patterned to the desired size and shape of the membrane on a silicon substrate ${ }^{2}$ (Step 1A-2A). Circular membranes are described from this point on for the sake of brevity. Undiluted PDMS was then spin-coated (PMW101, Headway Research) to barely cover the SU8 pillars (Step 3A). After curing the PDMS on a hotplate (TP781, Sigma Systems) at $110^{\circ} \mathrm{C}$ for $15 \mathrm{~min}$, the patterned PDMS base was peeled from the master and cut into individual pieces. Any membrane that may have formed over the SU8 pillars was removed by carefully peeling it off with a set of tweezers under a microscope (Step 6B, this can also be done after bonding). The PDMS surface that was formed on the silicon surface, having a pattern defined by the base of the posts, was used for suspending the membrane.

\footnotetext{
${ }^{2}$ All silicon substrates used in this report were (100), p-type, from Polishing Corporation of America.
} 


\subsubsection{Thin membrane bonding and surface treatment}

To allow the suspension of a thin PDMS membrane without it breaking, the silicon substrate was coated with diluted Teflon ${ }^{\circledR}$ solution as an adhesion reduction layer. Teflon ${ }^{\circledR}$ $\mathrm{AF}$, amorphous fluoropolymer resin in solution (601S1100-6), was purchased from Dupont, Inc. Fluorinert electronic liquid (FC-77) from 3M company was used to dilute the Teflon ${ }^{\circledR}$. After curing the Teflon ${ }^{\circledR}$ coating on a hot plate, diluted (with hexane) PDMS solution was spincoated (Step 1C); dilution ratios by weight of 1:15 for $\sim 490 \mathrm{~nm}$ and 1:75 for $100 \mathrm{~nm}$ thick membranes were used. The membrane was then cured on a hotplate at either $85^{\circ} \mathrm{C}$ or $90^{\circ} \mathrm{C}$ for $15 \mathrm{~min}$, and then treated with air plasma (Step $2 \mathrm{C}$ ); at the same time the structure from the previous step (base PDMS with an opening or the perforated membrane) was also treated with the air plasma. The two parts were then bonded (Step 3C). After peeling the bonded PDMS part from the handle silicon substrate, a freely suspended membrane was obtained over all the holes on the base PDMS structure (Step 4C). Optical images of some suspended PDMS membranes are shown in Fig. 3.

\subsubsection{Fabrication of silicon base and device assembly}

The silicon base for this device assembly served two purposes: (a) it prevented the deflection of the PDMS base structure during experiments, and (b) it allowed the membrane device to be handled easily (the device has to be flipped over to perform surface treatments necessary for certain experiments.) To fabricate a silicon base with a circular through hole, a silicon nitride layer was first grown (Step 1D) on a (100) silicon substrate using a low pressure

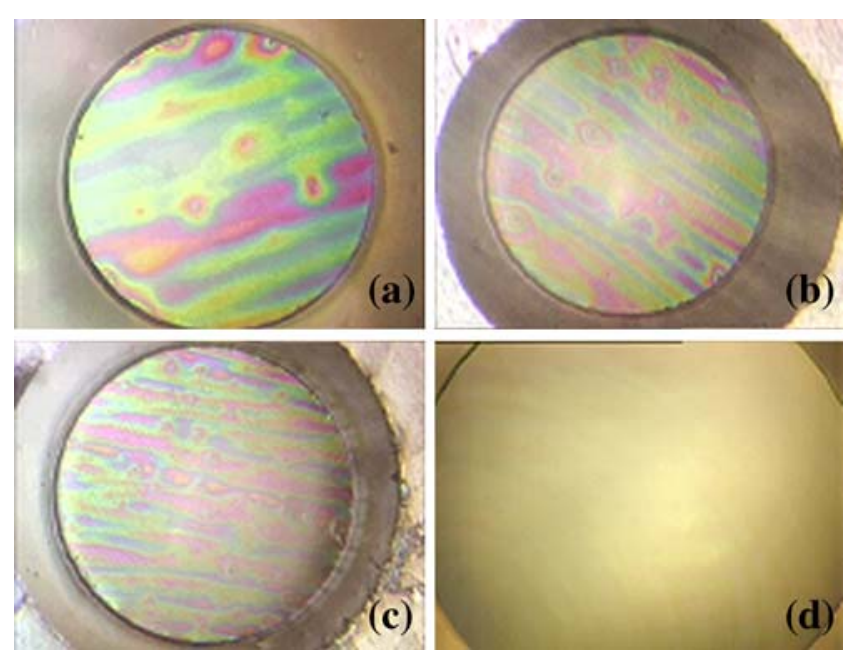

Fig. 3 Optical images of membrane devices with different diameters (a) $315 \mu \mathrm{m}$ (b) $488 \mu \mathrm{m}$ (c) $723 \mu \mathrm{m}$ (d) $491 \mu \mathrm{m}$. Membranes (a-c) are $492 \mathrm{~nm}$ thick while (d) is $3 \mu \mathrm{m} *$ thick (*Undiluted PDMS) chemical vapor deposition (LPCVD) furnace (BLUE, Process Technology Ltd.). The backside of the substrate was lithographically patterned to open large windows for $\mathrm{KOH}$ etching (Step 2D). These backside openings were designed to be much larger than the through-holes so as to not block light during experimentation/characterization of the membrane with an optical profilometer. The exposed silicon was etched in a $\mathrm{KOH}$ bath at $95^{\circ} \mathrm{C}$ until only a membrane with a thickness of approximately $50 \mu \mathrm{m}$ remains (Step 3D). Circular patterns, a little larger than the size of the PDMS membranes, were then opened from the front side using reactive ion etching (STS 340; Surface Technology Systems) after lithographically defining them (Step 4D). A hard baked photoresist (Shipley 1818, MicroChem Corp) was used as the etch mask for patterning the silicon nitride layer while a $200 \mathrm{~nm}$ thick chromium mask (deposited using e-beam evaporator (NRC-3117, Varian)) was used for etching the silicon membrane. After the silicon was etched through, the nitride layer was removed by dipping the substrate in concentrated hydrofluoric acid (Step 5D). The PDMS device was then bonded to the base silicon substrate (Step 6D). Since the membrane has to be aligned to the opening of the base silicon substrate, a drop of ethanol was placed on the silicon before the two parts were brought together. The PDMS device was then manipulated under an optical microscope until the membrane was aligned with the circular opening of the silicon base. Once the ethanol evaporated, a strong bond formed between the two parts. When a permanent bond was desired, the two bonding surfaces were treated with the air plasma before bonding. A thick PDMS structure with a $\sim 5 \mathrm{~mm}$ diameter through-hole was then placed on the PDMS side of the device assembly, aligning the structure hole with the membrane. This thick PDMS structure served as a seal for the liquid chamber during experiments.

\subsection{Fabrication of membrane with integrated patterns}

\subsubsection{Integration of patterns on top of the membrane}

Patterns integration on the PDMS membrane is important since it provides a mean to customize the membrane device. The patterns may be used, for example, as adhesion and growth sites for cells (Singhvi et al. 1994; Wang et al. 2002) or as a membrane deflection readout system (Zhao and Zhang 2005). To integrate micro/nanopatterns on topside of the membrane, the desired patterns were first created with photoresist on a silicon substrate. The substrate was coated with diluted Teflon ${ }^{\circledR}$ AF solution, (tridecafluoro-1,1,2,2-tetrahydrooctyl) trichlorosilane (TFOTS, Gelest), or a very thin layer of parylene (Parylene-C using PDS 2010 coater; Specialty Coating Systems) as an 

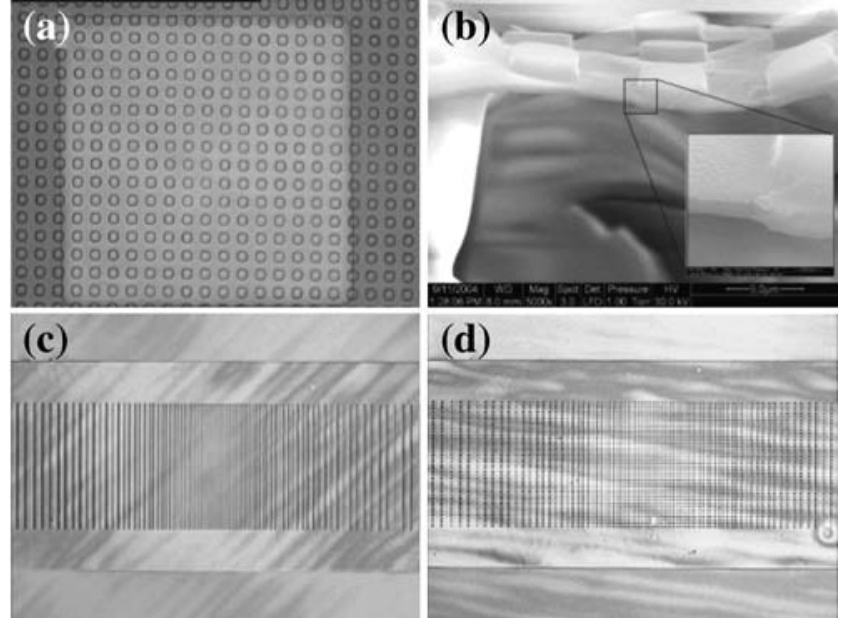

Fig. 4 PDMS membrane with integrated patterns. (a), (b) Patterns on top of suspended PDMS membrane (after suspension) and (c) Line patterns and (d) Dot patterns on the backside of the membrane (before suspension)

adhesion reduction layer. Diluted PDMS solution was then spin-coated to form a membrane on the substrate. A more detailed fabrication procedure was reported elsewhere (Thangawng and Lee 2004). When the patterned area of the membrane was suspended on another PDMS structure with an opening, a membrane with integrated micro/nano patterns was obtained (see Fig. 4).

\subsubsection{Integration of patterns on the backside of the membrane by soft molding}

It is possible to flip over the membrane with integrated patterns described above so that the patterns are on the backside of the membrane. However, it is necessary in many cases to directly suspend the patterns on the desired (e.g., back) side. Figure 5 shows the process of pattern integration on a flat membrane. First, the PDMS membrane was spin-coated to a desired thickness on a substrate coated with an adhesion reduction layer. A prefabricated mold (hard or soft) was then placed carefully on the PDMS membrane and pressure was applied, causing the mold to displace and penetrate the uncured PDMS to form the desired patterns. The membrane, with the mold and the load intact, was then heated to fully cure it. This imprinting pattern transfer technique is referred to as soft molding (Kim et al. 2001; Khang and Lee 2004). Excessive pressure can cause penetration through the film and thus an appropriate pressure must be carefully selected. Alternatively, a height difference in the patterns or the base substrate (as represented in Fig. 5) can be introduced to ensure that the membrane is not penetrated through.
Fig. 5 Pattern integration by soft-molding or imprinting
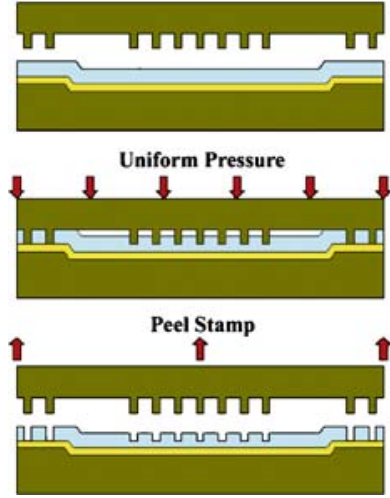

Figure 4(c) and (d) shows $3 \mu \mathrm{m}$ lines and dots patterns created using this technique.

\subsection{Suspension of ultra-thin membranes on an array} of micro features

As one might expect, the PDMS membrane thickness limits the size of the membrane that can be freely suspended without breaking. With the current fabrication protocol, membranes with thickness less than $150 \mathrm{~nm}$ can only be suspended over features smaller than $10 \mu \mathrm{m}$. If only one side of the membrane needs to be accessible or exposed, the membrane can be simply suspended over arrays of micro pillars or micro wells as demonstrated in Fig. 6. The membrane shown is $80 \mathrm{~nm}$ thick. On the other hand, if both sides of the membrane needed to be accessible, the process shown in Fig. 1 (with the suspension of a perforated membrane being a critical step) can be followed.
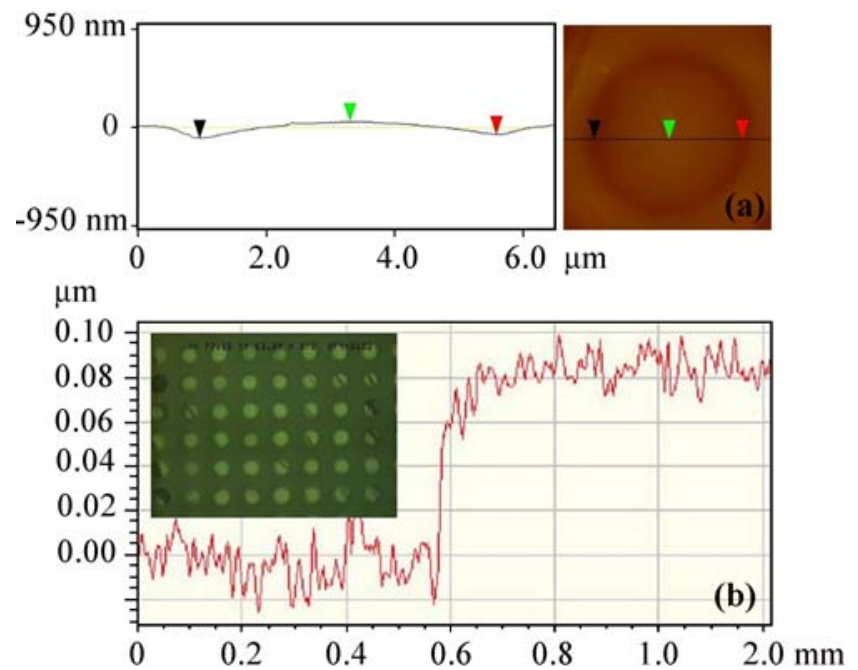

Fig. 6 (a) AFM scan of an ultra-thin PDMS membrane and (b) an optical profile showing the thickness of the membrane. The inset in (b) shows a light microscopic image of an array of suspended ultra-thin membrane 


\subsection{PDMS membrane formation on hydrophobic surfaces}

PDMS bonds very well (reversibly or irreversibly) to many smooth surfaces. In order to freely suspend a thin PDMS film, the strength of the adhesion between the cured PDMS membrane and the substrate needs to be dramatically reduced. A good way to achieve this is by coating the substrate with hydrophobic materials before the membrane is spin-coated. Photoresist (Shipley 1818; MicroChem Corp) and then separately parylene coating had been used as the adhesion reduction coating for membranes thicker than $1 \mu \mathrm{m}$. For membranes with submicron thicknesses, Teflon ${ }^{\circledR}$ AF 601S1-100-6 (Dupont) diluted with FC-77 flourinert solution (3M) was used as the adhesion reduction coating. Diluted PDMS solution can be readily spin-coated on relatively hydrophilic surfaces such as silicon and glass substrates. However, when a highly diluted PDMS solution (dilution ratio of 10 or above) was dispensed on a hydrophobic surface such as a Teflon ${ }^{\circledR}$-coated silicon substrate, the solution tends to roll off the surface due to the low surface tension force between the solution and the substrate. When the spinning was initiated, the solution that may have remained on the substrate simply rolled off and the membrane could not be formed. To prevent the diluted PDMS solution from rolling off, the outer edge of the hydrophobic surface was patterned to expose the bare silicon surface. This patterned ring (which is hydrophilic) acted as a retainer for the solution. The high surface tension force on the hydrophilic surface retains the solution on the substrate during spin-coating, allowing the formation of the desired membrane.

Patterning the hydrophobic surface (such as the Teflon ${ }^{\circledR}$ film itself) can be a challenge since many conventional

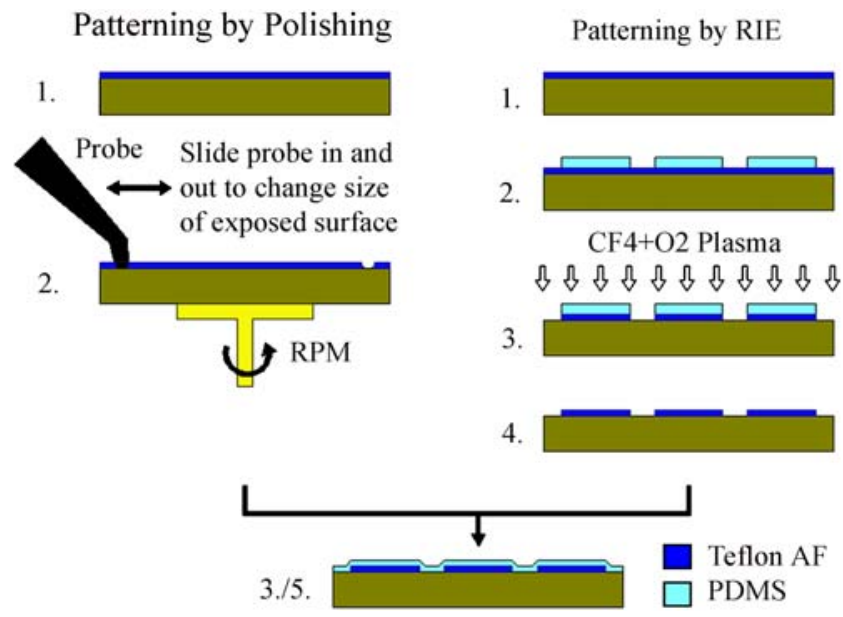

Fig. 7 Patterning Teflon ${ }^{\circledR}$ coating to form hydrophilic ring for fluid retention photoresists can not be spin-coated on it for the same reason the diluted PDMS solution can not be spin-coated. Two methods of patterning Teflon ${ }^{\circledR}$ were developed, see Fig. 7. The first uses mechanical polishing to remove the coating. The Teflon ${ }^{\circledR}$-coated substrate was spun at 2000 RPM and it was lightly touched at a desired location with a probe (clean blade or tweezers). In the process, the Teflon ${ }^{\circledR}$ coating was removed from the part of substrate that was in contact with the probe. Alternatively, a clean room wipe wetted with acetone or isopropyl alcohol can be used to remove the Teflon coating.

For the second patterning technique, a circular PDMS mask was placed on top of the Teflon ${ }^{\circledR}$ coating and the exposed Teflon ${ }^{\circledR}$ was etched in $\mathrm{CF}_{4}+\mathrm{O}_{2}$ plasma using the $\mathrm{RIE}$ machine. The PDMS mask was peeled off from the substrate after the etching was completed.

\section{Analytical equation for circular membrane loaded with a uniform pressure}

Both for simplicity and to obtain a uniform strain (Waters et al. 2001) on the membrane in the radial direction when loaded, a circular membrane was chosen. The geometry defining the membrane is depicted in Fig. 8. For larger membranes (a diameter of $300 \mu \mathrm{m}$ and larger), the bulge test was chosen for characterization. With the assumption that the membrane deflection is hemispherical in shape, the small deflection of the membrane is given by (Timoshenko et al. 1959; Pan et al. 1990)

$P=\frac{C_{1} t}{a^{2}} \sigma_{0} w_{0}+\frac{C_{2} f(v) t}{a^{4}} \frac{E}{1-v} w_{0}^{3}$

where $P$ is the uniform pressure applied to the membrane, $w_{0}$ is the maximum deflection measured at the center of the membrane, $a$ is the membrane radius, $t$ its thickness, $\sigma_{0}$ its residual stress, $E$ its Young's modulus, and $\nu$ its Poisson's ratio. The geometrical coefficients $C_{1}, C_{2}$, and $f(v)$ for circular membranes are 4, 2.67 and 1 respectively (Pan et al. 1990).

Equation 1 can be further simplified to calculate the residual stress on the membrane and the Young's modulus of the material. Dividing both sides of Eq. 1 by the deflection $w_{0}$ yields

$\frac{P}{w_{0}}=\frac{C_{1} t}{a^{2}} \sigma_{0}+\frac{C_{2} f(v) t}{a^{4}} \frac{E}{1-v} w_{0}^{2}$

which has the form $Y=A+B x^{2}$. A plot of $\frac{P}{w_{0}}$ versus $w_{0}^{2}$ gives a straight line and the slope can be used to calculate the Young's modulus while the intercept is used to calculate the residual stress. 
Fig. 8 (a) Schematic showing membrane geometry and loading condition, and (b) A typical screen shot from MicroXAM profiler output for data collection

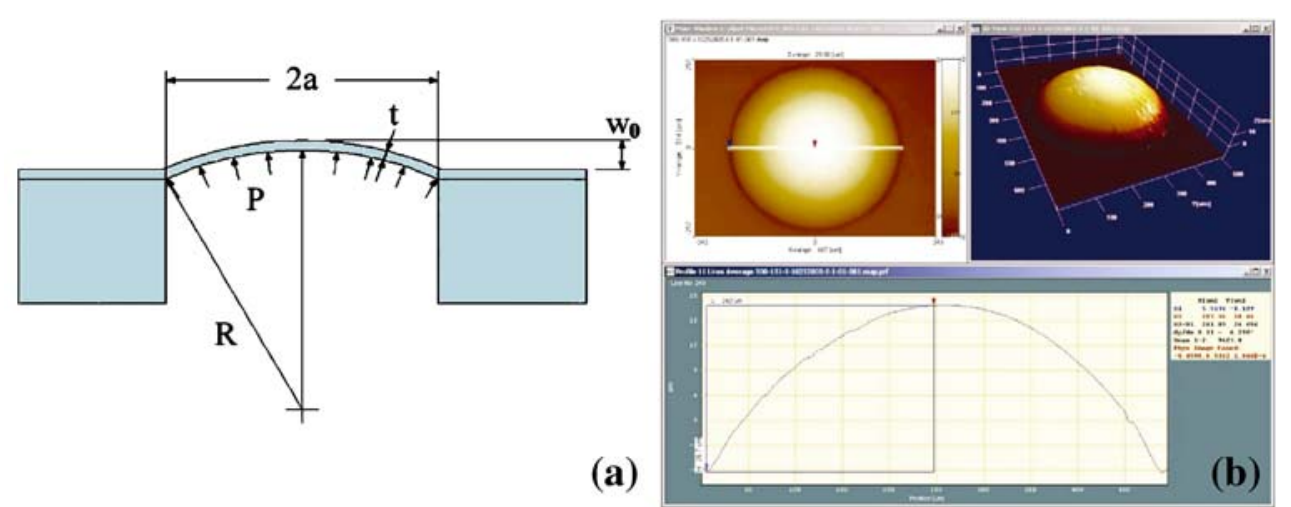

\section{Experiment: Bulge test for plain circular membrane}

\subsection{Fluidic cell design for bio/chemical experiments}

Since we are interested in simulating physiological conditions for future experiments, we fabricated a fluidic cell that is capable of delivering the media to one side of the membrane and also inflating the membrane (see Fig. 9). The fluidic cell has three different ports/openings: inlet, outlet, and device port. They are positioned at different heights. The membrane device was placed on the lowest port and the media was supplied from the highest port. This was critical for ensuring that full contact was maintained between the membrane and the media at all times, and it also eliminated the formation of bubbles inside the fluidic chamber. The membrane device was first placed over its port that has a PDMS seal on top. The device was then clamped from the top to ensure the chamber was leak tight. Another PDMS spacer was placed between the device and the clamp to prevent direct contact between the silicon base and the mechanical clamp to prevent the silicon base from breaking. The culture media (water for all characterization experiments when liquid media was used) was then supplied from the inlet port. The air-outlet port was maintained in the open position during the addition of the media to allow all bubbles to float away. The chamber was filled with media until it completely filled the outlet port. The air outlet port (mid level) was then plugged. The highest port of the fluidic cell was then connected to a U-tube manometer (with water as working fluid). An open U-tube manometer was build that allows precise control of the pressure. The system can be pressurized up to $10 \mathrm{kPa}$ using water. The water column height can be read accurately within $1 \mathrm{~mm}$, which corresponds to $\sim 10 \mathrm{~Pa}$. The initial membrane deflection was measured by scanning the membrane with an optical profilometer. The membrane was then pressurized incrementally by adding water in the manometer tube, which compressed the air trapped between the manometer and the fluidic cell. The corresponding membrane deflection for each pressure increment was scanned with the optical profiler. The change in water column height was also recorded for each measurement. The manometer acted as both the pressure sensor and the pressure transducer. A typical screen shot of the optical profilometer output for load-deflection data collection is shown in Fig. 8.

One critical feature of the experiment setup was the fact that the top of the membrane was dry, which was necessary for conducting measurements with the optical profilometer. Furthermore, it provided a good configuration for integrating
Fig. 9 Experimental setup for PDMS membrane characterization
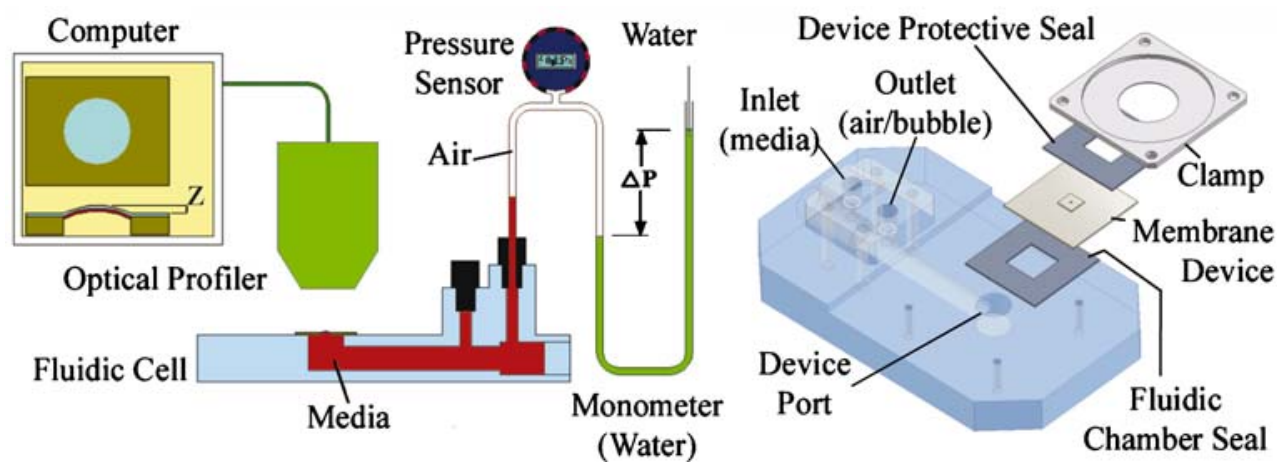
sensors for future development. A computer-controlled pump can be easily attached to the manometer to carry out a dynamic experiment. For a small deflection study, the manometer can be eliminated and the media inlet port can be used as a direct loading port, for both static and dynamic loading.

\section{Results and discussion}

Representative load-deflection experimental data are plotted in Fig. 10 for two different groups of devices (493 and $3,000 \mathrm{~nm}$ thick). For each group of devices, three different sizes (diameters), approximately 300, 500 and $750 \mu \mathrm{m}$, were tested. For clarity, data for only a single loadingunloading cycle is shown in the plots for each membrane. Up to four cycles of loading-unloading data from each membrane were used to calculate the residual stresses and effective biaxial modulus presented in Table 1. The membrane thickness of $492 \mathrm{~nm}$ is obtained by spin-coating a 1:15 diluted PDMS (in hexane), and the 3,000 nm thick membrane by spin-coating undiluted PDMS. Note the difference in the deflection values in Fig. 10 for membranes of similar diameters but with different thicknesses. As apparent from the plots, the membrane responses fit extremely well with the analytical model as given by Eq. 1. Within the range of deflections tested, the membrane response was elastic and the membranes can be reproducibly loaded and unloaded, virtually without any hysteresis. The membranes are extremely flexible due to the low Young's modulus and the thickness of the PDMS. As a comparison, a $492 \mathrm{~nm}$ thick PDMS membrane with a diameter of $287 \mu \mathrm{m}$ has similar load-deflection curves with a $55 \mathrm{~nm}$ thick $600 \mu \mathrm{m}$ (diameter) Au-polymer nanocomposite membrane reported by Jiang et al. (2004).

The biaxial modulus, $\frac{E}{(1-v)}$, are presented in Table 1 . Assuming $\nu=0.5$ for PDMS (Armanil et al. 1999), the average values of Young's modulus obtained from the experiments are 6.61 and $7.76 \mathrm{MPa}$ for undiluted and diluted PDMS films respectively. Obviously, these values are much higher than the normally reported values of 2.5 MPa. The Young's modulus values of bulk PDMS usually fall within $12 \mathrm{kPa}-2.50 \mathrm{MPa}$ (Tan et al. 2003; Brown et al. 2005; Armanil et al. 1999; Eddington et al. 2003; Gray et al. 2003), depending on the processing conditions. Since we obtained relatively similar values of Young's modulus for membranes fabricated with diluted and undiluted PDMS, the dilution of PDMS does not seems to be the primary cause of the much larger Young's modulus obtained. Rather, it was likely due to the fact that the membranes were formed by spincoating the PDMS solution at a high speed (6,000 RPM) for a relatively long time (up to $150 \mathrm{~s}$ ). The Young's modulus values reported in the literature are based on relatively thick (a few tens of microns to $2 \mathrm{~mm}$ ) substrates obtained by pouring the PDMS on a flat base substrate. We speculate that the polymer chains are prestretched in our spin-coating process while they are in a relaxed state for the ones prepared by pouring on a flat substrate without any rotation. The fact that there is a $2.51 \mathrm{MPa}$ difference in the experimentally obtained biaxial modulus between the undiluted and diluted PDMS also supports this mechanism. Undiluted PDMS is extremely viscous compare to the diluted PDMS and it would require much more centripetal force to untangle the polymer chains. The processing temperature can also
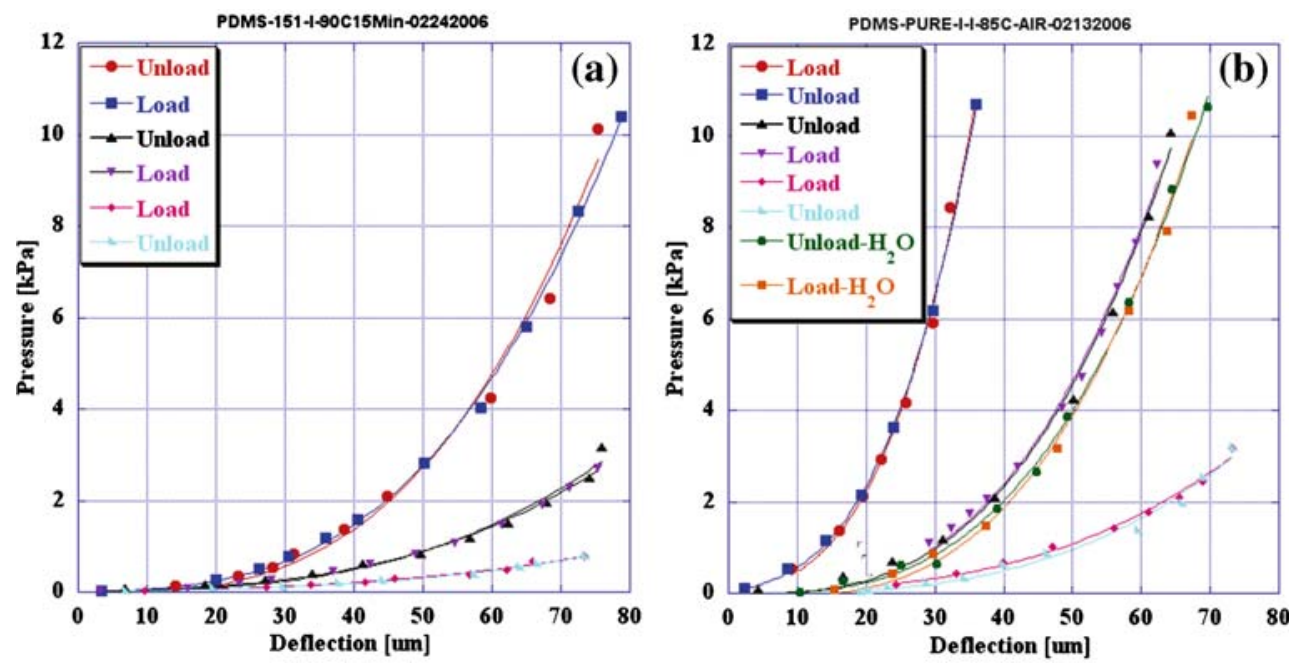

Fig. 10 Load-deflection curves for membranes with different sizes and thicknesses (a) $492 \mathrm{~nm}$ thick with a diameter of $[\bullet, \mathbf{\square}] 315.1 \mu \mathrm{m},[\mathbf{\Delta}, \boldsymbol{\nabla}]$ $488.63 \mu \mathrm{m},[\bullet, \mathbf{\bullet}] 731.2 \mu \mathrm{m}$ (b) $3,000 \mathrm{~nm}$ thick with a diameter of $[\bullet, \mathbf{\square}] 287.3 \mu \mathrm{m},[\boldsymbol{\Delta}, \boldsymbol{\nabla}, \bullet, \mathbf{\bullet}] 491.32 \mu \mathrm{m},[\bullet, \mathbf{\Delta}] 722.7 \mu \mathrm{m}$ 
Table 1 Calculated residual stress and Young's modulus values for PDMS membranes

\begin{tabular}{|c|c|c|c|c|c|}
\hline Sample ID & Membrane diameter $[\mu \mathrm{m}]$ & Membrane thickness [nm] & Process temperature $\left[{ }^{\circ} \mathrm{C}\right]$ & Residual stress [MPa] & $E /(1-v)[\mathrm{MPa}]$ \\
\hline $300-\mathrm{I}$ & 287.3 & 3,000 (Pure) & $85(15 \mathrm{~min})$ & 0.072 & 10.40 \\
\hline 500-I & 491.3 & 3,000 (Pure) & 85 (15 min) & 0.050 & 15.60 \\
\hline $500-\mathrm{I}-\mathrm{H}_{2} \mathrm{O}$ & 491.3 & 3,000 (Pure) & $85(15 \mathrm{~min})$ & -0.005 & 14.72 \\
\hline $750-\mathrm{I}$ & 722.7 & 3,000 (Pure) & $85(15 \mathrm{~min})$ & 0.048 & 15.06 \\
\hline \multirow[t]{3}{*}{ 1000-I } & \multirow[t]{3}{*}{986.1} & \multirow[t]{3}{*}{ 3,000 (Pure) } & $85(15 \mathrm{~min})$ & 0.047 & 10.19 \\
\hline & & & Average & 0.042 & 13.22 \\
\hline & & & standard deviation & 0.028 & 2.64 \\
\hline 300-I-15:1 & 315.1 & 492 (Diluted) & $90(15 \mathrm{~min})$ & 0.040 & 9.59 \\
\hline 500-I-15:1 & 488.6 & 492 (Diluted) & $90(15 \mathrm{~min})$ & 0.162 & 13.87 \\
\hline 500-II-15:1 & 488.0 & 492 (Diluted) & $90(15 \mathrm{~min})$ & 0.138 & 19.64 \\
\hline $750-\mathrm{I}-15: 1$ & 731.2 & 492 (Diluted) & $90(15 \mathrm{~min})$ & 0.177 & 15.66 \\
\hline \multirow[t]{3}{*}{ 750-II-15:1 } & \multirow[t]{3}{*}{722.9} & \multirow[t]{3}{*}{492 (Diluted) } & $90(15 \mathrm{~min})$ & 0.176 & 18.80 \\
\hline & & & Average & 0.129 & 15.51 \\
\hline & & & standard deviation & 0.057 & 4.05 \\
\hline
\end{tabular}

have a major effect on the Young's modulus of the materials (Eddington et al. 2003).

Other conditions that might have contributed significantly to the large Young's modulus obtained are the flatness of the membrane and the uniformity of the membrane thickness. When the PDMS membranes were peeled from the substrate during the fabrication process, ridges were formed if the membrane gets folded (small, step-wise folding), and multiple folding may occur during the process. These ridges can become permanent if the membrane is exposed to $\mathrm{O}_{2}$ plasma. The ridge formation was more pronounced in thinner membranes, as one would expect, and also when the membrane was exposed to the $\mathrm{O}_{2}$ plasma for a longer period. Optical images showing four different membrane devices are shown in Fig. 3. A close inspection of these images revealed that number of ridges increases as the membrane is more flexible. The alternating colors of the membrane in the images also suggest that the membranes were not truly flat. We attribute this to the non-uniform coating of the Teflon ${ }^{\circledR}$ layer and dirt particles that were introduced during the preparation of the solution. For future membrane fabrication, the non-uniformity in the adhesion reduction layer may possibly be eliminated by using a vapordeposited adhesion reduction layer instead of spin-coating a solution-based agent. The calculated effective Young's modulus may vary from device to device due to the individual handling during the fabrication process and the geometry of the membrane but it is evident from the data obtained that each individual device exhibited reproducibility during experiments and that they can be used effectively with proper calibration, much like any other mechanical devices used for precision sensing/measurements.

The majority of data presented here are from experiments where the membranes were inflated by air. Although PDMS is reported to be one of the most gas permeable polymers (Charati and Stern 1998), we did not observe any change in the pressure inside the fluidic chamber for the duration of our experiments, which suggested that the gas permeation through the membrane can be neglected for such lengths of time. The membrane response was found to be the same when the chamber was filled with liquid, specifically water. Data for both air and water as the pressure medium of the same membrane with a diameter of 491.3 $\mu \mathrm{m}$ are plotted in Fig. 10(b). The difference in the membrane deflections of the two experiments was due to the initial deflection of the membrane in the water filled experiment. This initial deflection resulted from the built-up hydrostatic pressure as the membrane sat lower than the highest level of water in the fluidic cell. This hydrostatic pressure was approximately $0.30 \mathrm{kPa}$ and it closely corresponds to the shift shown in Fig. 10(b). The slopes of the plot $\frac{p}{w}$ vs. $w^{2}$ (not shown), which were used to calculate the Young's modulus of the material, are essentially the same for the membrane tested with air or water as the pressure media inside the fluidic chamber.

\section{Conclusion}

In this paper, we demonstrated the extreme flexibility of thin PDMS membranes. The ease of fabrication of both plain membranes and those having integrated micro/nanopatterns, and PDMS' biocompatibility and its biologically relevant mechanical properties make the membrane system a suitable candidate as an interface to study biological systems in the micro and nano environments. The micropattern integration is being exploited to fabricate a membrane that has spatially varying stiffness and it is being used to investigate cellsubstrate interactions. These membrane systems are also envisioned to be used as platforms for studying mechanical 
properties of very soft materials such as cell secreted basement membrane proteins, and also as bio/chemical sensors.

Acknowledgement Funding for this project was provided by NIH (HL075217) and NSF (BES-0134551).

\section{References}

D. Armani1, C. Liu 1, N. Aluru, in 12th IEEE International Conference on Micro Electro Mechanical Systems (MEMS '99), pp. 222-227, 1999

N.Q. Balaban, U.S. Schwarz, D. Riveline, P. Goichberg, G. Tzur, I. Sabanay, D. Mahalu, S. Safran, A. Bershadsky, L. Addadi, B. Geiger, Nat. Cell Biol. 3, 466-472 (2001)

I.B. Bischofs, U.S. Schwarz, Proc. Natl. Acad. Sci. U.S.A. 100(16), 9274-9279 (2003)

I.B. Bischofs, S.A. Safran, U.S. Schwarz, Phys. Rev., E 69, 021911 (2004)

X.Q. Brown, K. Ookawa, J.Y. Wong, Biomaterials 26, 3123-3129 (2005)

K. Burton, D.L. Taylor, Nature 450-454 (1997)

S.G. Charati, S.A. Stern, Macromolecules 31, 5529-5535 (1998)

M.N. De Silva, R. Desai, D.J. Odde, Biomedical Devices 6(3), 219222 (2004)

O. du Roure, A. Saez, A. Buguin, R.H. Austin, P. Chavrier, P. Siberzan, B. Ladoux, Proc. Natl. Acad. Sci. U.S.A. 102(7), 2390-2395 (2005)

D.T. Eddington, W.C. Crone, D.J. Beebe, in 7th International Conference on Miniaturized Chemical and Biochemical Analysts Systems, Squaw Valley, CA, pp. 1089-1092, 2003

D.S. Gray, J. Tien, C.S. Chen, J. Biomed. Mater. Res. 66A, 605-614 (2003)

A.K. Harris, P. Wild, D. Stopak, Science 208(4440), 177-179 (1980)
C. Jiang, S. Markutsya, Y. Pikus, V.V. Tsukruk, Nat. Mater. 3, 721728 (2004)

D.-Y. Khang, H.H. Lee, Langmuir 20, 2445-2448 (2004)

Y.S. Kim, K.Y. Suh, H.H. Lee, Appl. Phys. Lett. 79(14), 2285-2287 (2001)

C.-M. Lo, H.-B. Wang, M. Dembo, Y.-1. Wang, Biophys. J. 79, 144$152(2000)$

J.C. Lotters y, W. Olthuis, P.H. Veltink, P. Bergveld, J. Micromechanics Microengineering 7, 145-147 (1997)

E. Ostuni, R. Kane, C.S. Chen, D.E. Ingber, G.M. Whitesides, Langmuir 16, 7811-7819 (2000)

J.Y. Pan, P. Lin, F. Maseeh, S.D. Senturia, in Tech. Digest IEEE SolidState Sensors Workshop, pp. 70-73, 1990

S.L. Peterson, A. McDonald, P.L. Gourley, D.Y. Sasaki, J. Biomed. Mater. Res., Part A 72(1), 10-18 (2005)

R. Singhvi, A. Kumar, G.P. Lopez, G.N. Stephanopoulos, D.I. Wang, G.M. Whitesides, D.E. Ingber, Science 264, 696-698 (1994)

J.L. Tan, J. Tien, D.M. Pirone, D.S. Gray, K. Bhadriraju, C.S. Chen, Proc. Natl. Acad. Sci. U.S.A. 100(4), 1484-1489 (2003)

A.L. Thangawng, J. Lee, in Proceedings of IMECE04, 2004 International Mechanical Engineering Congress, Anaheim, CA, 13-20 November 2004

A.L. Thangawng, M.A. Swartz, M.R. Glucksberg, R.S. Ruoff, Small 3, 132-138 (2007)

S. Timoshenko, S. Woinowsky-Krieger, Theory of plates and shells (McGraw-Hill, New York, 1959)

H.-B. Wang, M. Dembo, S.K. Hanks, Y.-1. Wang, Proc. Natl. Acad. Sci. U.S.A. 98(20), 11295-11300 (2001)

N. Wang, E. Ostuni, G.M. Whitesides, D.E. Ingber, Cell Motil. Cytoskelet. 52, 97-106 (2002)

C.M. Waters, M.R. Glucksberg, E.P. Lautenschlager, C.-W. Lee, R.M. Van Matre, R.J. Warp, U. Savla, K.E. Healy, B. Moran, D.G. Castner, J.P. Bearinger, J. Appl. Physiol. 91, 1600-1610 (2001)

Y. Zhao, X. Zhang, Mater. Res. Soc. Symp. Proc. 845, AA5.10.1AA5.10.6 (2005) 